\title{
EchoGéo
}

3 | 2007

La nouvelle donne en Amérique latine

\section{Entretien avec Thierry Sanjuan}

\section{Olivier Ninot}

\section{OpenEdition}

Journals

Édition électronique

URL : https://journals.openedition.org/echogeo/1822

DOI : 10.4000/echogeo.1822

ISSN : 1963-1197

\section{Éditeur}

Pôle de recherche pour l'organisation et la diffusion de l'information géographique (CNRS UMR 8586)

\section{Référence électronique}

Olivier Ninot, «Entretien avec Thierry Sanjuan », EchoGéo [En ligne], 3 | 2007, mis en ligne le 22 février 2008, consulté le 31 juillet 2021. URL : http://journals.openedition.org/echogeo/1822 ; DOI : https:// doi.org/10.4000/echogeo.1822

Ce document a été généré automatiquement le 31 juillet 2021.

EchoGéo est mis à disposition selon les termes de la licence Creative Commons Attribution - Pas d'Utilisation Commerciale - Pas de Modification 4.0 International (CC BY-NC-ND) 


\title{
Entretien avec Thierry Sanjuan
}

\author{
Olivier Ninot
}

1 Le dictionnaire de la Chine contemporaine est paru en novembre 2006 aux éditions Armand Colin. Il a été rédigé par un collectif de 88 auteurs, spécialistes des sciences sociales, réunis sous la direction de Thierry Sanjuan, professeur de géographie à l'Université de Paris 1 - Panthéon-Sorbonne. Le dictionnaire de la Chine contemporaine a deux objectifs : «donner à comprendre la complexité de l'actuelle transition chinoise et dessiner la silhouette, sur le plan intérieur comme dans ses actions extérieures, d'un pays qui est déjà l'un des pôles majeurs de l'espace mondial de ce nouveau siècle » (p. IX). Il répond à ce double objectif par une liste de 300 articles qui éclairent, dans des analyses croisées, les grandes questions et évolutions sociales, culturelles, politiques et économiques de la Chine contemporaine. Les articles retenus répondent aux critères d'analyse des sciences sociales (histoire, politique, droit, économie, géographie, urbanisme, sociologie, anthropologie, culture, etc.) et prennent également en compte les spécificités chinoises. Par exemple, si l'on y trouve bien l'article «religion», la définition du terme et les renvois qui y sont associés permettent de découvrir l'ambiguïté et le caractère problématique de cette notion en Chine. Le dictionnaire comprend également une introduction de 6 pages, une chronologie de 2 pages, un index, une bibliographie, ainsi que 9 cartes et plans hors texte, soit au total 304 pages riches et denses.

2 Le dictionnaire de la Chine contemporaine offre plusieurs modes et niveaux de lecture différents. Un premier est celui d'une lecture tous azimuts. On recherche, à partir de l'index, les définitions données pour les mots, noms propres, lieux, événements ou symboles que l'on connaît ou reconnaît et qui soit attisent la curiosité, soit renvoient à l'imagerie et aux idées reçues que tout un chacun possède sur la Chine. Ce sont par exemple « Tian'anmen », « Longue Marche », « Mao Zedong », « Révolution culturelle », "Shanghai », etc. Mais l'index et les renvois à la fin des articles invitent rapidement à d'autres modes de lectures. Il faut se laisser guider, aller vers des entrées inconnues ou surprenantes (par exemple pêle-mêle, "bureau des lettres et des visites", " enregistrement de la résidence », "organisation de masse ", ou encore "obésité ", « sexualité ", « rock », etc.) pour parvenir à une information experte, qui s'avère peutêtre destinée prioritairement aux lecteurs avertis, en tout cas un peu au fait des réalités 
chinoises. Enfin, un mode de lecture plus systématique et plus méthodique permet, à partir d'un article et en suivant les renvois, d'adopter une approche thématique et de couvrir complètement une question ou une notion particulière. Ainsi, l'article «ville » renvoie à « centre commercial », « entreprise d'État », « espace public », « immobilier », « logement», "patrimoine», " urbanisme», "villes et campagnes»; ces articles renvoyant eux-mêmes à d'autres tels que «enregistrement de la résidence ", " mégalopole ", " migrations intérieures ", " parcs et jardins ", « résidents ", etc.

3 Les géographes chercheront en vain l'article «territoire» ou encore celui de " nation ${ }^{1}$ ", entrées et concepts peut-être peu utiles à la compréhension de la Chine contemporaine, mais trouveront de nombreux articles renvoyant à l'organisation de l'espace national: des cardinalités associées à des projets de développement et d'aménagement (Ouest, Nord-Est), des noms de lieux et de régions (Xinjiang, delta de la rivière des Perles, etc.), des notions et catégories spatiales (local, région frontalière, etc.). Le local est un thème récurrent, une clef de compréhension évidente d'une donnée de base de la Chine contemporaine qui est son extrême morcellement : le pays est immense, sa diversité et sa complexité sur les plans de la topographie, des cadres administratifs, du peuplement, des langues, etc. imposent le local comme échelle d'approche privilégiée. Á l'opposé scalaire, d'autres articles, également nombreux, réfèrent aux relations extérieures de la Chine, abordant à la fois les questions des frontières, de la diplomatie, du commerce international.

4 Après avoir parcouru ce dictionnaire en plusieurs sens, on connaît un peu mieux la Chine, ou moins mal. Les deux grands objectifs de ce dictionnaire sont atteints: l'éclairage des mutations en cours dans la chine contemporaine en dessine une silhouette complexe, parfois incertaine, tant les évolutions sont rapides et les bouleversements profonds, en dépit d'inerties fortes ça et là. Car les mutations chinoises ne peuvent être simplement interprétées en termes de modernisation, encore moins d'occidentalisation ou d' " ajustement » aux modèles issus de la globalisation. La Chine ne se banalise pas, nous dit T. Sanjuan en introduction.

5 Au final, par les articles retenus, les thèmes et les questions traités, la pertinence, la richesse et l'actualité des définitions proposées, ce dictionnaire ne témoigne peut-être pas tant de la modernité de la Chine que de la modernité de ses spécialistes.

Compte rendu d'entretien avec Thierry Sanjuan

6 ÉchoGéo

Quelle est l'origine de ce dictionnaire ? D'où en est venue l'idée ?

T.S. L'idée a été lancée par l'ancienne responsable de la géographie des éditions Armand Colin. Á l'époque, la plupart des ouvrages consacrés à la Chine ne proposaient que des visions partielles du pays. Il y avait donc un espace éditorial à occuper, les dictionnaires étaient et sont encore à la mode, et les éditions Armand Colin affichaient un goût marqué pour ce genre d'exercice. Dès le départ, l'idée fut de confier ce projet à un collectif d'auteurs sous la responsabilité de coordonnateurs thématiques et d'un directeur de publication. Dès le départ également, il ne s'agissait pas de faire un dictionnaire général, sans problématique particulière, mais bien un dictionnaire de la Chine d'aujourd'hui. Entre un dictionnaire de la Chine et un dictionnaire de la Chine contemporaine, l'adjectif change tout !

\section{7 ÉchoGéo}

Le collectif d'auteurs réunit 88 spécialistes de la Chine issus des sciences sociales. Comment fut-il constitué? 
T.S.

La construction du savoir sur la Chine n'est plus aujourd'hui un domaine réservé à de stricts sinologues. Elle incombe à des spécialistes de disciplines différentes. La connaissance de la Chine contemporaine est donc nécessairement fragmentée et seule la réunion d'un collectif pouvait permettre d'en proposer une vision globale.

9 J'ai choisi les coordonnateurs, qui eux-mêmes m'ont proposé des auteurs. Le choix des auteurs a répondu à deux critères assez simples. Le premier fut que tous devaient être francophones. C'était indispensable pour comprendre parfaitement les problématiques qui fixent les lignes directrices de ce dictionnaire, et pour proposer des contributions mobilisant les termes français des concepts des sciences sociales utiles à la compréhension de la Chine contemporaine. Le second fut que ce collectif n'avait pas l'ambition d'être exhaustif ou représentatif de la sinologie française. Nous sommes partis des entrées retenues pour ce dictionnaire et pas des auteurs. Ceux qui ont participé ont donc été sollicités en fonction de ce qu'ils pouvaient apporter à cet ouvrage particulier qu'est un dictionnaire de la Chine contemporaine.

Par ailleurs, je dois souligner qu'il n'y a eu aucune ambiguïté relative au rôle de coordinateur qui m’a été confié. Tout le monde a joué le jeu, les multiples et fréquentes réunions se sont déroulées dans une très bonne entente, ce qui a facilité à la fois la rédaction et la coordination. Les délais ont d'ailleurs été tenus : le dictionnaire a vu le jour en deux ans et demi.

10 ÉchoGéo

Comment s'est faite la sélection des articles ? Trois cents articles, c'est beaucoup et peu à la fois.

11 T.S.

Tout a été fait en commun : la sélection des articles, les relectures et corrections, etc. Il manque peut-être quelques articles, mais le contrat a globalement été rempli, le dictionnaire est fidèle au projet initial. Bien sûr, la liste des entrées pourrait être allongée, complétée, révisée touts les ans voire plus souvent, tellement les mutations que connaît la Chine sont rapides. Le terme "blogue " est un bon exemple. Le développement des «blogues » (blogs) est très récent (moins de 5 ans) en Chine, mais il a pris une telle ampleur (16 millions de blogueurs en 2005) qu'il est apparu nécessaire de faire figurer le terme dans notre dictionnaire. Finalement, le défi posé par la sélection des entrées était celui du projet éditorial dont rend compte le titre de l'ouvrage : contemporain, qu'est ce que cela veut dire? Est-ce que c'est après 1949 ? Non, il n'y a pas de date butoir permettant de définir ce qui est contemporain. En fait, ce qui est contemporain, ce qui justifie le choix des articles du dictionnaire, c'est tout ce qui fait sens dans la Chine d'aujourd'hui. Ensuite, par la sélection des articles, nous avons eu l'ambition de montrer des processus, des mutations, des structures sociales, économiques, territoriales, etc., et de faire en sorte que la Chine apparaisse moins étrangère, plus accessible, à la lecture de ce dictionnaire.

\section{2 ÉchoGéo}

Justement, en lisant ce dictionnaire, on ressent que la rédaction de chaque article a été alimentée par une connaissance fine, pratique et concrète des phénomènes expliqués, acquise notamment par la recherche de terrain.

13 T.S.

C'était un impératif! Il nous a en effet paru indispensable que ce dictionnaire s'appuie sur la connaissance de terrain qu'ont les auteurs. C'est l'une des originalités de cet 
ouvrage et il s'agit, en fait, d'un phénomène générationnel. La plupart des auteurs ont entre 40 et 55 ans. Ils forment une génération de spécialistes arrivant à "maturité scientifique » qui n'a connu que la Chine des réformes et qui a pu mener des recherches de terrain. Pour les chercheurs qui ont été formés dans les années 1960 ou 1970, c'était impossible: il n'y avait plus de statistiques en Chine, plus d'université, plus de recherche. L'anthropologie, par exemple, était une discipline interdite! Aujourd'hui, les conditions de travail restent difficiles, mais il est au moins possible de faire de la recherche de terrain en Chine. Bon nombre de thèses sont soutenues chaque année par de jeunes chercheurs de moins de trente ans, dirigés par quelques uns des auteurs de ce dictionnaire.

\section{4 ÉchoGéo}

De nombreuses entrées sont consacrées aux relations entre la chine et le monde (migrations internationales, la Chine et tel ou tel pays ou groupe de pays, etc.). Or, une partie de la fascination que la Chine suscite, une partie des inquiétudes nourries à son égard, tient à ses relations internationales.

T.S.

C'est l'une des problématiques développées dans le dictionnaire; problématique nécessaire mais pas prioritaire. Le dictionnaire est celui de la Chine contemporaine et pas celui de la Chine dans le monde, même si aujourd'hui c'est vrai que l'ouverture de la Chine est une problématique importante et un phénomène qui contribue à la fois à la rendre un peu moins mystérieuse ou moins étrangère, tout en alimentant les phantasmes sur la puissance chinoise. Car, plus que jamais la Chine fascine, elle est à la mode.

EchoGéo

Si la Chine connaît des bouleversements profonds affectant ses secteurs productifs, ses modes de vie, ses représentations, on ne peut pour autant voir dans ses mutations une simple modernisation. La trajectoire que suit la Chine contemporaine est proprement chinoise, elle n'a rien d'une occidentalisation, mais a-t-elle vocation à se diffuser, à se proposer en modèle?

17 T.S.

Non. La Chine de Mao était un modèle. Aujourd'hui, on n'a plus, en Chine, la volonté d'imposer un modèle chinois. Il existe une volonté nationaliste mais pas celle d'imposer un modèle chinois. La trajectoire de la Chine se présente plutôt comme une alternative : il y a dans la culture chinoise, dans l'évolution du pays, dans les façons de faire, les modes de vie, etc. des propositions alternatives à celles existant en Asie, en Occident ou ailleurs. Sans doute, la Chine fournira-t-elle des modèles (la première ville du monde entièrement écologique est en train d'être construite en Chine), mais elle ne sera pas toute entière un modèle. On n'est pas aujourd'hui dans une logique de pôle qui diffuse mais dans celle d'un ensemble qui produit de l'innovation pour lui-même.

Propos recueillis par Olivier Ninot. 


\section{NOTES}

1. Mais on trouve « nationalisme » et « nationalité ».

\section{AUTEUR}

\section{OLIVIER NINOT}

Le dictionnaire de la Chine contemporaine a reçu le Prix Ernest Lémonon 2007 de l'Académie des Sciences Morales et Politiques.

Thierry Sanjuan (tsanjuan@univ-paris1.fr) vient également de diriger un numéro de la revue Hérodote et de publier l'« Atlas de la Chine » aux éditions Autrement «Chine, nouveaux enjeux géopolitiques ", Hérodote $n^{\circ} 125$, second trimestre, éditions La Découverte, 2007, 190 p. et Atlas de la Chine : Les mutations accélérées, éditions Autrement, 2007, 80 p. 